\title{
Films of chitosan and natural modified hydroxyapatite as effective UV-protecting, biocompatible and antibacterial wound dressings
}

\author{
Carla S. Cunha a,b, Pedro J. Castro ${ }^{\text {a }}$, Sérgio C. Sousa ${ }^{\text {a }}$, Robert C. Pullar ${ }^{\mathrm{c}}$, David M. Tobaldi ${ }^{\mathrm{c}}$, \\ Clara Piccirillo ${ }^{\mathrm{d}, *}$, Maria M. Pintado ${ }^{\text {a }}$ \\ a CBQF - Centro de Biotecnologia e Química Fina - Laboratório Associado, Escola Superior de Biotecnologia, Universidade Católica Portuguesa/Porto, Porto, Portugal \\ b Department of Chemical Engineering, University of Porto, Porto, Portugal \\ c Department of Materials and Ceramic Engineering and CICECO-Aveiro Institute of Materials, University of Aveiro, Campus Universitário de Santiago, $3810-193$ Aveiro, Portugal \\ d CNR NANOTEC, Institute of Nanotechnology, Campus Ecoteckne, Lecce, Italy
}

\section{A R T I C L E I N F O}

\section{Article history:}

Received 19 March 2020

Received in revised form 30 April 2020

Accepted 11 May 2020

Available online 19 May 2020

\section{Keywords:}

Chitosan

Hydroxyapatite

Wound dressing

UV protection

Antibacterial

\begin{abstract}
A B S T R A C T
Chitosan is a natural polysaccharide widely used in biomedicine, for instance for wound dressing. Hydroxyapatite is a very bioactive calcium phosphate which, if modified with an appropriate element (iron Fe), can also have UVabsorbing properties. In this work, we report the study of films of chitosan incorporated with iron-modified hydroxyapatite of natural origin (from cod fish bones); this combination led to an innovative chitosan-based material with excellent and advanced functional properties. The films showed very high UV absorption (Ultraviolet Protection Factor (UPF) value higher than 50). This is the first time that a chitosan-based material has shown such high UV protection properties. The films also showed to be non-cytotoxic, and possessed antimicrobial activity towards both Gram-positive and negative strains. Their mechanical properties, optimised with an experimental design approach, confirmed their potential use as multifunctional wound dressing, capable of reducing bacterial infections and, at the same time, protecting from UV light.
\end{abstract}

(c) 2020 Elsevier B.V. All rights reserved.

\section{Introduction}

Chitosan (CS) is a natural polysaccharide obtained by deacetylation from chitin, a major component of crustacean shells [1]. Due to many interesting properties, chitosan is a widely used compound in biomedicine. CS is highly biocompatible, non-toxic and biodegradable, and shows antibacterial properties towards several strains [2]. Moreover, CS is also suitable for wound dressings, with reported effectiveness in wound healing due to its active role in various phases of the healing process [3]. Because of this, CS-based wound dressings have been prepared in different forms, including hydrogels, membranes and films.

Ultraviolet (UV) protection is an additional functionality which, if present in a wound dressing, would be beneficial for patients. The dangers associated with UV radiation to human skin are well known [4,5], as it can cause severe damage and/or lead to lethal diseases, as well as suppressing the immune system. Protection against UV radiation is desirable for damaged skin, wounds and burnt skin [6]. Indeed studies showed that, while exposure to small doses of UVC radiation can promote wound healing [7], prolonged exposure to UV radiation can have a negative effect on the skin. However, CS does not absorb in the UV (200-400 nm); to do so, chitosan has to be combined with other

\footnotetext{
* Corresponding author.

E-mail address: clara.piccirillo@nanotec.cnr.it (C. Piccirillo).
}

compounds which can act as sunscreens. There are reports of composite materials consisting of CS and both organic and inorganic sunscreens, and results showed the potential of CS-based composites, as materials with UV-absorbing properties were successfully prepared [8-10].

Hydroxyapatite $\left(\mathrm{Ca}_{10}\left(\mathrm{PO}_{4}\right)_{6}(\mathrm{OH})_{2}\right.$, $\left.\mathrm{HAp}\right)$ is a calcium phosphate compound also employed in biomedicine. HAp is the main component of human bone, used to fabricate bone replacements/implants, as it replicates closely the composition and behaviour of the original bone [11]. HAp is highly biocompatible and non-toxic. The use of HAp as sunscreen has been investigated, and the presence of specific dopants in the HAp structure (i.e. zinc, manganese), or other compounds in a multiphasic material, can give good UV-absorbing materials [12]. Iron is also an interesting dopant for UV absorbance [13].

Many reports exist of composite materials based on HAp and CS [14], but only one considers a composite for sunscreen applications [15]. Although this material showed limited UV-absorbing performance, and absorbance was quite high for $\lambda<250 \mathrm{~nm}$ (UVC), it decreased significantly for higher wavelengths. This is crucial, as UVC is mostly absorbed by the atmosphere, and the harmful forms are UVB (290-320 nm) and UVA (320-400 nm) on the Earth's surface [16].

Here, we report the development of a composite of CS and UVabsorbing HAp, made as a film, using commercial CS, and HAp of marine origin (derived from waste fish bones). HAp was prepared by calcining bones pretreated in an iron-containing solution, forming a powder 
consisting of $\mathrm{HAp}, \alpha-\mathrm{Fe}_{2} \mathrm{O}_{3}$ and a mixed iron-calcium-phosphate [17]. Previous studies performed by the authors showed strong absorbance in the whole UV range of this composite material due to $\alpha-\mathrm{Fe}_{2} \mathrm{O}_{3}$ optical properties and the synergistic effect with the phosphates.

The objective of this work is to develop a chitosan-based material in which its excellent biomedical properties can be combined with the UVabsorbing properties of the HAp-based powder. Our hypothesis is that the combination of chitosan and modified HAp results in a material with excellent UV-absorbing properties, as the incorporation of the powder in the chitosan matrix does not compromise its optical behaviour; at the same time, the coatings retain the mechanical, antibacterial, wound healing and non-cytotoxic CS functionalities. The composite films were characterised, and their mechanical properties were optimised, using an experimental design approach. In addition, functional properties such as the Ultraviolet Protection Factor (UPF), antibacterial activity and cytotoxicity were measured.

\section{Materials and methods}

\subsection{HAp powder preparation}

The preparation of the HAp-based powder was previously described $[13,17]$. Briefly, bones were treated in a basic solution containing excess $\mathrm{FeCl}_{2}$ (Sigma Aldrich, $99 \%$ purity) for $5 \mathrm{~h}$, then dried at $45-50{ }^{\circ} \mathrm{C}$ overnight, and calcined at $700{ }^{\circ} \mathrm{C}$ (heating $5{ }^{\circ} \mathrm{C} / \mathrm{min}, 1 \mathrm{~h}$ dwell). This powder (named FeHAp) underwent high energy ball milling, previous studies [17] showing that this created a powder with more uniform and smaller particle size.

\subsection{Film preparation}

CS films were obtained using the casting/solvent evaporation method. CS (Sigma Aldrich, medium molecular weight, 190,000-310,000 Da, > 99.0\%, deacetylation grade 75-85\%) was dissolved under vigorous stirring in glacial acetic acid (Sigma Aldrich, > $99.85 \%)(1 \% \mathrm{v} / \mathrm{v})$ at $25^{\circ} \mathrm{C} / 24 \mathrm{~h}$ (concentration $\left.=1 \% \mathrm{w} / \mathrm{v}\right)$. After complete dissolution, FeHAp powder was added in various concentrations.

Glycerol (Sigma Aldrich, >99\%) was added to the solution as a plasticiser, to obtain a film with appropriate mechanical properties. The suspension (total volume of $60 \mathrm{~mL}$ ) was left stirring on a magnetic stirrer/hot plate at $40^{\circ} \mathrm{C}$ for $30 \mathrm{~min}$. Then this was treated ultrasonically with a high intensity ultrasonic processor (Sonics vibra-cell VCX 750 Watt, Sonics \& Materials, Newtown, CT, USA), with an amplitude of $70 \%$, for $30 \mathrm{~min}$; this step was necessary to separate the aggregated FeHAp particles, as well as to remove air bubbles from the suspension. The solution was cooled with an ice bath to avoid overheating and chitosan degradation. The solution was then stirred for $10 \mathrm{~min}$ at $40{ }^{\circ} \mathrm{C}$, poured into a glass plate $(10 \times 50 \mathrm{~cm})$ and dried at $25^{\circ} \mathrm{C}$ for $72 \mathrm{~h}$.

Table 1 reports the list of the samples prepared with this methodology. Some preliminary characterisation was performed on these films, to determine the best preparation conditions and to assess whether these systems showed UV-absorbing properties.

\subsection{Characterisation of the films and functional properties tests}

Analyses to assess the morphology of the films and their UV absorbing properties were performed on the samples listed in Table 1, as detailed in Section 2.3.1.

\subsubsection{Morphology and UV-absorbing properties}

The morphology of the films (CS-FeHAp) was studied with scanning electron microscopy (SEM, Hitachi S-4100, JP), on films coated with carbon. Energy-dispersive X-ray spectroscopy (EDS) analysis was also performed to determine the films' composition, and confirm the incorporation of FeHAp powder into the CS matrix.

\section{Table 1}

List of the films prepared. During the casting solution, the chitosan concentration was kept constant, while powder and glycerol concentrations were changed.

\begin{tabular}{llll}
\hline Sample name & \multicolumn{2}{c}{ Concentration of the casting solution $(\% \mathrm{w} / \mathrm{v})$} \\
\cline { 2 - 4 } & HAp & FeHAp & Glycerol \\
\hline 1 & - & 1 & 2.5 \\
2 & - & 1 & 3.0 \\
3 & - & 1 & 3.5 \\
4 & - & 1 & 4.0 \\
5 & - & 1 & 4.5 \\
6 & - & 2 & 2.5 \\
7 & - & 2 & 3.0 \\
8 & - & 2 & 3.5 \\
9 & - & 2 & 4.0 \\
10 & - & 2 & 4.5 \\
11 & - & 3 & 2.5 \\
12 & - & 3 & 3.0 \\
13 & - & 3 & 3.5 \\
14 & - & 3 & 4.0 \\
15 & - & 3 & 4.5 \\
CS & - & - & 2.5 \\
CS-HAp & 2 & - & 2.5 \\
\hline
\end{tabular}

An initial UV screening was performed using a Shimadzu UV 3100 (JP) spectrometer, with a $\mathrm{BaSO}_{4}$ integrating sphere and $\mathrm{BaSO}_{4}$ as a white reference material. Spectra were acquired in the reflectance mode in the UV-vis range ( $250-825 \mathrm{~nm}, 0.2 \mathrm{~nm}$ step size). The data were converted into pseudo-absorption spectra $(\alpha)$ by the KubelkaMunk function:

$\alpha=\frac{(1-R)^{2}}{2 R}$

These first tests were performed to see whether the powder retained UV-absorbing behaviour after incorporation into the chitosan matrix [17].

The Ultraviolet Protection Factor (UPF) was measured for some selected films with a Labsphere UV-2000F ultraviolet transmittance analyser (Labsphere Inc., NH, USA), following the AU/NZS 4399 protocol for both measurement and sample preparation [18]. UPF measurements were carried out in UV from 280 to $400 \mathrm{~nm}$ - the value was calculated according to:

$U P F=\frac{\sum_{290}^{400} E(\lambda) S(\lambda)}{\sum_{290}^{400} E(\lambda) S(\lambda) T(\lambda)} * 100$

where $E(\lambda)=$ CIE reference erythema dose spectrum, $S(\lambda)=$ sunlight radiation intensity distribution and $\mathrm{T}(\lambda)=$ diffuse transmittance spectrum (\%). Three replicates were prepared for each film, with samples placed against the flush transmission port opening in the sphere, and four different points were measured for each sample. Using the same apparatus, the critical wavelength value $\lambda_{\text {crit }}$ was also calculated (wavelength corresponding to an absorbance equal to $90 \%$ of the total):

$\int_{290}^{\lambda_{\text {crit }}} A(\lambda) d \lambda=0.9 * \int_{290}^{400} A(\lambda) d \lambda$

where $A(\lambda)=$ absorbance at each wavelength.

To optimise the mechanical behaviour of the films, an experimental design approach was employed, as detailed in the sections below.

\subsubsection{Experimental design}

Experimental design and statistical analysis was performed to obtain the best possible formulation for the CS-FeHAp films, using JMP®10 (SAS institute, UK) software. Three design factors were selected as independent variables - glycerol concentration (plasticiser), FeHAp concentration (active ingredient) and film thickness. Properties described in Sections 2.3.3-2.3.7 were set as dependent variables (responses). 
These tests were performed on films with selected FeHAp and glycerol composition, based on the results of the previous characterisation described in Section 2.3.1. Every response test was performed in triplicate for each sample. A model design was executed, and independent variables that influenced the behaviour of the evaluated dependent variables were selected for the elaboration of the predictive statistic model.

To verify that the response values obtained were normally distributed, the Shapiro-Wilk test was used. One sample $t$-test was used to verify if there were statistically significant differences between the predictive models and experimental values. Experimental values were obtained from the preparation of three selected samples of new batches of films. The mean values for each batch were compared with the predicted values in the model.

\subsubsection{Mechanical properties}

Mechanical properties of CS-FeHAp films were measured using a texturometer TA.XTplus Texture Analyser (Stable Micro Systems, UK) with a $5 \mathrm{~kg}$ load cell, using ASTM protocols, as reported in literature $[19,20]$. Several properties were assessed, as described below. For all tests, samples with dimension of $70 \times 20 \mathrm{~mm}$ were used.

Film burst strength (BS, g) and distance at burst (DB, $\mathrm{mm}$ ) were determined using a $5 \mathrm{~mm}$ stainless steel cylinder tip. The tip was moved onto the film at $0.5 \mathrm{~mm} \mathrm{~s}^{-1}$, until rupture, and the force $(\mathrm{g})$ recorded as a function of deformation ( $\mathrm{mm}$ ). The maximum force of the peak $=$ $\mathrm{BS}$, while the distance to the peak $=\mathrm{BD}$.

BS and BD were also assessed after the films had been in contact with a liquid. Films were placed into Falcon tubes containing $30 \mathrm{~mL}$ of PBS solution $(0.9 \% \mathrm{w} / \mathrm{v})$, adjusted to sweat $\mathrm{pH}(6.6)$ and pre-heated at $37{ }^{\circ} \mathrm{C}$, and left immersed for $1 \mathrm{~h}$.

Tensile strength (TS, MPa), elongation break (EB, \%), Young's modulus (YM, MPa) and strain energy (SE, MPa) were measured using a miniature tensile grip probe. Film strips of $2 \times 7 \mathrm{~cm}$ were held between 2 clamps, and pulled by the top clamp at a rate of $0.5 \mathrm{~mm} \mathrm{~s}^{-1}$. The force and elongation were measured when the film broke, while TS represents the maximum tensile stress borne before breaking. For all properties, the values are the average of 4 analyses.

\subsubsection{Film thickness}

Thickness of the films was measured by calibrated digital Verniergauge caliper micrometer (SE 784EC Digital Caliper; SE Tools, Lapeer, MI, USA), measured at three points for each film, and the average value determined.

\subsubsection{Water uptake and erosion}

Water-uptake (WU \%, also known as swelling degree) was determined by placing the films $(30 \times 30 \mathrm{~mm})$ in contact with $5 \mathrm{~mL}$ of saline solution $(\mathrm{NaCl} 0.9 \% \mathrm{w} / \mathrm{v})$, adjusted to sweat $\mathrm{pH}$ 6.6. Weight was registered at 120 mins, and WU calculated considering the percentage difference in weight in comparison to the original weight. Based on this, the value of WU/time (\%/min) was calculated, by dividing the WU by the corresponding time:

$\frac{\text { Water uptake }}{\text { time }}=\frac{\text { Max } W U}{\text { time } \max W U}$

After immersion in solution, hydrated samples were placed in an oven at $60^{\circ} \mathrm{C}$ for $24 \mathrm{~h}$, and weight variation recorded to determine erosion. Erosion (E, \%) is the amount of weight lost by the film during contact with saline solution.

Disintegration of the films was assessed by leaving them in saline solution for $24 \mathrm{~h}$ at $37^{\circ} \mathrm{C}$, after which their integrity was checked.

\subsubsection{Film colour}

Colour of the films was measured by CM-700d spectrophotometer (Konica-Minolta, JP), calibrated with a standard white plate $\left(\mathrm{L}^{*}=\right.$ $97.59, \mathrm{a}^{*}=0.07, \mathrm{~b}^{*}=1.89$ ). The L-a-b method, also known as CIELAB, was used, the colour difference between each film and the reference film being calculated by:

$\Delta E=\left[\left(\Delta L^{*}\right)^{2}+\left(\Delta a^{*}\right)^{2}+\left(\Delta b^{*}\right)^{2}\right]^{1 / 2}$

where $\Delta \mathrm{L}^{*}, \Delta \mathrm{a}^{*}$ and $\Delta \mathrm{b}^{*}$ are the difference of each coordinate between the tested CS-FeHAp film and the reference one (CS). A $5 \times 5 \mathrm{~cm}$ film was used, and each film measured 3 times. The values were calculated as an average, with the corresponding standard deviation.

\subsubsection{Ex-vivo bioadhesive strength}

Bioadhesive strength was assessed by measuring the peak detachment force (PDF), performed using TA.XT2plus texture analyser equipment (see Section 2.3.3). Frozen pig skin extracted from pig ear was used as model tissue, after thawing. The protocol employed was that reported in literature, with some modifications [21]. Briefly, pig ear skin $(70 \times 20 \mathrm{~mm})$ was fixed to the lower platform of the texture analyser with suitable adhesive tape. The film was fixed with a suitable adhesive tape to a probe in the top part of the equipment, which was aligned to the lower support. During measurement, $10 \mu \mathrm{L}$ of simulated sweat fluid was evenly spread on the surface of the tissue. The film was lowered at a speed of $1.0 \mathrm{~mm} \mathrm{~s}^{-1}$ to contact the tissue (epidermal part of the pig skin) at $1 \mathrm{~N}$ force for 15 min contact time, then withdrawn at $1.0 \mathrm{~mm} \mathrm{~s}^{-1}$ to $10 \mathrm{~mm}$ distance. PDF values $(\mathrm{N})$ were expressed as the mean of three replicates.

For the film with optimised mechanical properties, additional tests of porosity, cytotoxicity and antibacterial activity were performed, as described below.

\subsubsection{Porosity}

The porosity of the films was assessed with the protocol reported by Khorasani et al. [22]: the films were placed in dry ethanol until saturation (about $60 \mathrm{~min}$ ), and their weights before and after the immersion were measured. The porosity (\%) was calculated according to eq. 6 :

$P=\frac{W_{f}-W_{i}}{\rho V} \times 100$

where $W_{i}$ and $W_{f}$ are the weight before and after the immersion, $\rho$ is the density of ethanol and $V$ is the volume of the film.

\subsubsection{Cytotoxicity of the films}

Cytotoxicity of CS-FeHAp films was assessed with an adapted ISO10993-5 standard method protocol [23]. Human keratinocyte (HaCat) cell lines (Cell Lines Service, DE) were tested, using the 2,3bis-(2-methoxy-4-nitro-5-sulfophenyl)-2H-tetrazolium-5-

carboxanilide salt (XTT) colourimetric assay (Thermo Fisher Scientific). This assessed cell viability as a function of cell number based on metabolic activity. HaCat cells were seeded at a density of $1 \times 10^{5}$ cells per well, in a 6-well microplate, and allowed to adhere. After $24 \mathrm{~h}$, the media was removed and the cells washed with sterile PBS to remove dead cells. Following this, the medium with CS-FeHAp or CS films $\left(2 \mathrm{~cm}^{2}\right)$ was added. After $24 \mathrm{~h}, 25 \mu \mathrm{L}$ of XTT solution was added to each well, and the cells incubated in the dark for $2 \mathrm{~h}$. Then the films were removed from the solution, and absorbance measured at $485 \mathrm{~nm}$ by microplate reader (FLUOstar, OPTIMA, BMG Labtech, DE). Cells in simple culture media, without contact with any sample, were used as a positive control.

All assays were performed in quintuplicate, and statistical analysis was performed using SPSS 20 software (SPSS, Chicago, IL, USA). Normality of the data was determined by Shapiro-Wilk, and differences were assessed by $t$-test, with $5 \%$ significance.

\subsubsection{Antimicrobial activity of the films}

Antimicrobial activity was assessed by viable cell count assay, using protocols already reported in literature [24], with some modifications. 
Briefly, liquid cultures of selected microorganisms were grown overnight at $37^{\circ} \mathrm{C}$ in Mueller-Hinton broth, and the bacterial concentration adjusted to approximately $10^{+6}$ colony forming units (CFU) per $\mathrm{mL}$. $8.5 \mathrm{~g} \cdot \mathrm{L}^{-1}$ sodium chloride and $1.0 \mathrm{~g} \cdot \mathrm{L}^{-1}$ peptone water solutions were used for dilution.

$1.3 \mathrm{~cm}$ diameter film discs (CS film control and CS-FeHAp film) were sterilised under UV light for $10 \mathrm{~min}$ on each side. The film discs were placed in sterile tubes, and $200 \mu \mathrm{L}$ of liquid inoculum were placed over each disc. The films were left in contact with the inoculum for 2, 4, and $8 \mathrm{~h}$ at $37^{\circ} \mathrm{C}$. At each sampling time, $1.8 \mathrm{~mL}$ of dilution solution was added to each tube, and successive dilutions were prepared. Aliquots $(20 \mu \mathrm{L})$ of each diluted solution were planted on Muller-Hinton agar (Biokar Diagnostics, France) and, after incubation at $37^{\circ} \mathrm{C}$, colonies were counted. Each experiment was performed in triplicate, with statistical analysis performed as described in Section 2.3.8.

The tested microorganisms were a Gram-negative bacteria, Escherichia coli (ATCC 25922), a Gram-positive bacteria, methicillinresistant Staphyloccocus aureus (MRSA, CCUG 60578), and yeast, Candida albicans ( $\mathrm{CBQF}$ isolate).

\section{Results and discussion}

3.1. Powder incorporation: effect on morphology and UV-absorbing properties

Full characterisation of the FeHAp powder has already been published, see Teixeira et al. [17] for the detailed composition (approximately 56 wt\% $\mathrm{HAp}, 39 \mathrm{wt} \% \mathrm{Ca}{ }_{9} \mathrm{FeH}\left(\mathrm{PO}_{4}\right)_{7}$, and $\left.5 \mathrm{wt} \% \alpha-\mathrm{Fe}_{2} \mathrm{O}_{3}\right)$.

Preliminary tests were performed to assess the suitable concentration ranges of both glycerol and FeHAp powder. For glycerol, a content $<2.5 \%$ did not lead to obvious changes in film properties, while for values $>4.5 \%$ the films were too sticky, and difficult to handle. For the powder it was difficult to have a uniform incorporation for concentrations $>3 \%$. Based on this, films with the concentrations listed in Table 1 were studied.

In Fig. 1(a), photographs of FeHAp films are reported - they showed the same characteristic colour of the powder, with colour intensity

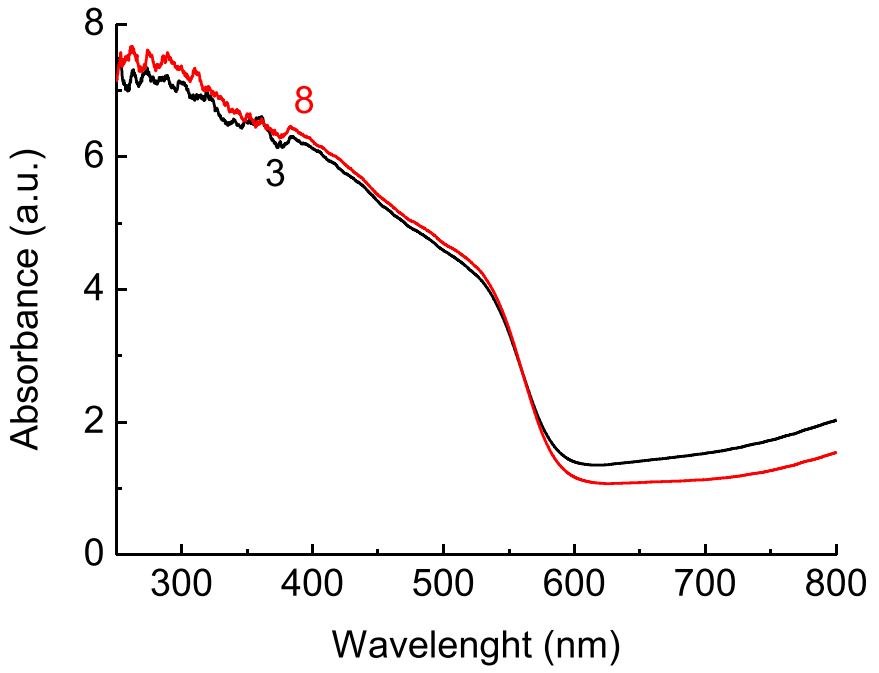

Fig. 2. UV spectra for films 3 and 8.

increasing for greater powder concentration, confirming its incorporation into the CS matrix.

SEM shows the morphology of films with incorporated powder. Fig. 1(b) shows sample $\mathbf{6}$ - the film is compact, with no visible cracks or other defects. With higher magnification (10,000 X, Fig. 1(c)), in some areas particles a few hundred nanometers in diameter, corresponding to FeHAp, can be observed; in other areas, however, the particles tend to aggregate into larger structures, a few microns in size. Overall, the powder seems uniformly incorporated into the CS matrix. Fig. 1(d) shows the EDS spectrum of this film, and peaks corresponding to $\mathrm{Ca}, \mathrm{P}$ and $\mathrm{Fe}$ are present. The micrographs of the other samples presented similar characteristics (data not shown).

In Fig. 2, the UV absorbance spectra of samples $\mathbf{3}$ and $\mathbf{8}$ are shown as examples ( 1 and $2 \% \mathrm{w} / \mathrm{v}$ FeHAp, respectively). Both films show the absorption profile of the FeHAp powder previously observed, indicating that the incorporation of the powder into the CS matrix does not alter
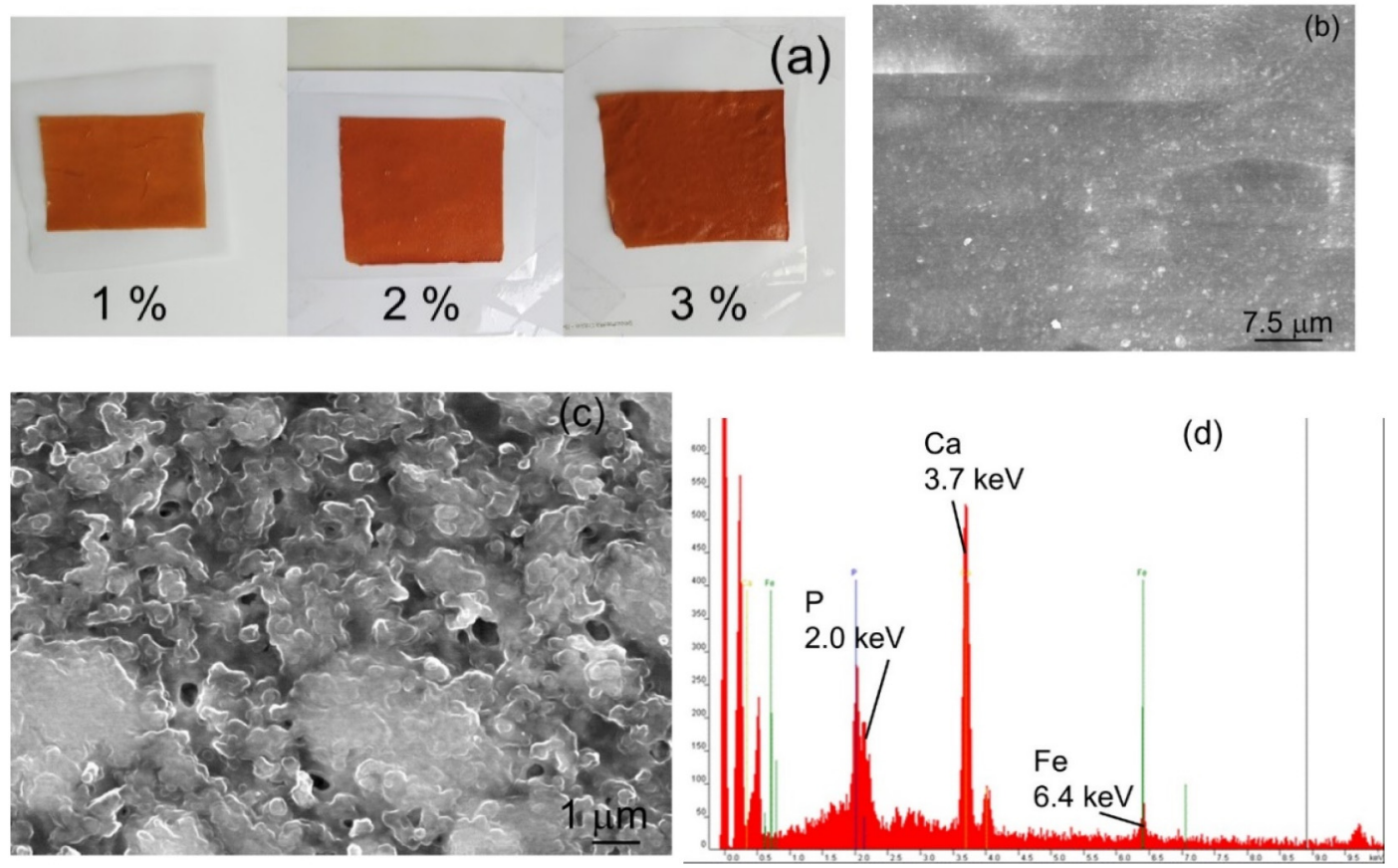

Fig. 1. (a) Pictures of the films with 1, 2 and 3 wt\% of FeHAp; (b) and (c) SEM micrographs of sample 6; (d) EDS spectrum of the same sample. 
Table 2

UPV values, critical wavelength $\lambda_{\text {crit }}$ and percentage transmittance for some selected CSbased films.

\begin{tabular}{llllll}
\hline Sample & UPF value & UPF Rating & $\lambda_{\text {crit }}(\mathrm{nm})$ & $\mathrm{T}_{\mathrm{UVA}}(\%)$ & $\mathrm{T}_{\mathrm{UVB}}(\%)$ \\
\hline 3 & 518 & Excellent & 386 & 0.93 & 0.12 \\
8 & 1996 & Excellent & 388 & 0.07 & 0.05 \\
13 & 2000 & Excellent & 389 & 0.05 & 0.05 \\
CS & 1.33 & Very low & 381 & 83.91 & 74.11 \\
CS-HAp & 6.33 & Very low & 381 & 25.17 & 14.88 \\
\hline
\end{tabular}

its UV-absorbing properties. This feature was already observed when FeHAp was incorporated into a non-chitosan cream [17]; the data reported here show that the powder has the capability of retaining its properties also in a solid medium. This indicates that the interactions between the powder and the chitosan matrix do not significantly affect the powder's optical properties.

Sample 8 shows a slightly higher absorbance in the UV interval $(\lambda<400 \mathrm{~nm})$, which is reasonable. It must be remembered, however, that these measurements are not quantitative, giving just an indication of the absorption profile and possible qualitative differences between various samples.

To quantitatively assess UV protection properties, UPF values were measured, and results for selected samples are reported in Table 2.

The films made of just CS, or CS and unmodified HAp, have relatively low UPF values - about 1 and 6, respectively. According to the AS/NZS standard, this corresponds to very low protection. The incorporation of FeHAp powder led to significant increases in UPF values, and all three FeHAp-containing films have a UPF value $>50$, corresponding to the highest protection. With $1 \% \mathrm{w} / \mathrm{v}$ FeHAp (sample 3), a UPF value $>500$ was observed, increasing even more with $2 \% \mathrm{w} / \mathrm{v}$ (sample 8) to almost 2000. Higher powder concentration ( $3 \% \mathrm{w} / \mathrm{v}$, sample 13), however, did not lead to improved UV protection - transmittance was very low in both UVA and UVB, but no significant differences were seen between samples 8 and 13. Regarding the critical wavelength $\lambda_{\text {crit }}$, all samples incorporated with FeHAp showed a value $>370 \mathrm{~nm}$, indicating that an effective protection is provided for the whole UV interval.

Films with the same FeHAp concentrations, but different glycerol content, showed similar results; this is reasonable, since glycerol does not absorb in the UV. Possible differences in the homogeneity in the films did not have any effect on the UV absorption, but only on their mechanical/plastic behaviour. Similarly, small differences in thickness and colour were also not determinant. However, both of these parameters were considered in the optimisation of the mechanical properties (see next section).

\subsection{Optimisation of the mechanical properties: Experimental design}

Considering the excellent UV protection properties of the films, an experimental design approach was implemented to optimise their mechanical properties. The list of samples used in the study is shown in Table 3; the name of the sample is indicated as ED, to avoid confusion with previous samples. The samples were chosen considering suitable ranges of composition for both FeHAp and glycerol concentrations. Specifically, for FeHAp 1, 1.5 and 2\% w/v were considered, as films with higher content (i.e. $3 \% \mathrm{w} / \mathrm{v}$ ) exhibited a stronger colour without significant improvement in UPF. For glycerol, on the other hand, concentrations of $2.5,3.5$ and $4.5 \% \mathrm{w} / \mathrm{v}$ were used. Samples with different compositions were not considered further in the study. For the experimental design, FeHAp and glycerol content, as well as film thickness, were set as independent variables. The dependent variables were measured experimentally, as detailed above. All results are shown in Table 3 and Fig. 3.

For some parameters, the correlation between either glycerol or FeHAp content and the measured value is very evident; e.g., higher glycerol concentration led to lower BS values (Fig. 3b), regardless of FeHAp content. BS was also measured after immersion in solution for $1 \mathrm{~h}$ (curves in the lower part of the graph), which caused a significant (8-10 times) BS decrease. For YM, TS and SE, in the majority of cases a decrease was observed with higher glycerol content (Fig. 3e, f and h, respectively); this correlation between mechanical properties and plasticiser content was previously reported for other natural polymers films $[25,26]$.

An increase in EB with higher glycerol content would be expected. However, experimental data (Fig. $3 \mathrm{~g}$ ) show that this is not always the case. This anomaly could be explained considering all interactions taking place between the powder particles incorporated into the polymeric structure. Such interactions are difficult to predict, due to the complexity of the system, and can lead to unusual/unexpected properties. HAp is the main component of the FeHAp powder; literature reports interactions between ionic groups present in both CS and HAp (amino and phosphate groups, respectively) [27]. Similarly, glycerol OH groups can interact with CS amino groups and $\mathrm{HAp} \mathrm{Ca}^{2+}$ ions [28]. Therefore, it can be seen that such a range of different interactions can significantly affect the characteristics of the films. Similar anomalous behaviour was previously reported for chitosan-based composite coatings - in fact, literature reports different effects on the EB value in coatings containing organic stabilisers or inorganic powders, and an increase in the concentration of these components did not lead to a linear increase/decrease $[29,30]$. Indeed, other properties of the films such as PDF, DB and WU/ time (Fig. 3a, c and d) did not show a clear correlation with either glycerol or HAp content.

However, film erosion (Fig. 3i) increases proportionally with glycerol content, while decreasing with higher FeHAp concentration. This could be explained by the powder making the film more compact, due to the strong electrostatic interactions between the different functional groups mentioned above.

Based on these experimental design data, the variables PDF, BS, DB, $\mathrm{YM}, \mathrm{EB}$ and SE were maximised, while WU/time, $\mathrm{E}$ and $\Delta \mathrm{E}$ were

Table 3

Factors and responses of the experimental design.

\begin{tabular}{|c|c|c|c|c|c|c|c|c|c|c|c|c|c|c|c|}
\hline \multicolumn{4}{|c|}{ Independent Variables } & \multicolumn{12}{|c|}{ Responses (dependent variables) } \\
\hline $\begin{array}{l}\text { Samples } \\
\text { (n) }\end{array}$ & $\begin{array}{l}\text { [FeHAp] } \\
(\%, \mathrm{w} / \mathrm{v})\end{array}$ & $\begin{array}{l}\text { [Glycerol] } \\
(\%, \mathrm{w} / \mathrm{v})\end{array}$ & $\begin{array}{l}\text { Thickness } \\
(\mathrm{mm})\end{array}$ & $\begin{array}{l}\text { PDF } \\
(\mathrm{N})\end{array}$ & $\begin{array}{l}\text { BS } \\
(\mathrm{g})\end{array}$ & $\begin{array}{l}\text { BS } 1 \mathrm{~h} \\
(\mathrm{~g})\end{array}$ & $\begin{array}{l}\mathrm{DB} \\
(\mathrm{mm})\end{array}$ & $\begin{array}{l}\text { DB } 1 \mathrm{~h} \\
(\mathrm{~mm})\end{array}$ & $\begin{array}{l}\text { Erosion } \\
(\%)\end{array}$ & $\begin{array}{l}\text { WU/time } \\
\left(\% \cdot \mathrm{min}^{-1}\right)\end{array}$ & $\begin{array}{l}\mathrm{YM} \\
(\mathrm{MPa})\end{array}$ & $\begin{array}{l}\text { TS } \\
(\mathrm{MPa})\end{array}$ & $\mathrm{EB}(\%)$ & $\begin{array}{l}\mathrm{SE} \\
(\mathrm{MPa})\end{array}$ & $\Delta \mathrm{E}$ \\
\hline ED1 & 1.0 & 2.5 & 0.07 & 0.019 & 193.44 & 18.95 & 4.15 & 1.31 & 60.79 & 2.15 & 0.0140 & 1.7185 & 125.55 & 121.39 & 57.62 \\
\hline ED2 & 1.0 & 3.5 & 0.06 & 0.023 & 180.68 & 33.36 & 3.16 & 3.09 & 66.51 & 1.85 & 0.0180 & 1.9316 & 112.18 & 129.83 & 57.82 \\
\hline ED3 & 1.0 & 4.5 & 0.07 & 0.034 & 119.62 & 20.63 & 3.57 & 2.65 & 71.92 & 2.34 & 0.0084 & 1.1884 & 126.12 & 80.96 & 56.27 \\
\hline ED4 & 1.5 & 2.5 & 0.07 & 0.022 & 272.25 & 44.54 & 3.10 & 2.78 & 52.28 & 2.12 & 0.0432 & 2.3550 & 72.22 & 126.04 & 57.78 \\
\hline ED5a & 1.5 & 3.5 & 0.08 & 0.037 & 192.40 & 22.39 & 3.70 & 2.52 & 64.06 & 1.85 & 0.0246 & 1.7173 & 112.57 & 131.80 & 58.35 \\
\hline ED5b & 1.5 & 3.5 & 0.08 & 0.037 & 192.73 & 22.42 & 3.91 & 2.54 & 60.97 & 1.87 & 0.0244 & 1.7276 & 112.98 & 131.61 & 58.38 \\
\hline ED6 & 1.5 & 4.5 & 0.11 & 0.037 & 96.01 & 15.61 & 4.23 & 2.05 & 67.03 & 2.19 & 0.0093 & 1.0892 & 138.63 & 91.60 & 56.67 \\
\hline ED7 & 2.0 & 2.5 & 0.07 & 0.036 & 211.92 & 41.45 & 2.58 & 2.30 & 49.49 & 1.10 & 0.0416 & 2.3884 & 98.01 & 217.89 & 57.65 \\
\hline ED8 & 2.0 & 3.5 & 0.07 & 0.042 & 212.13 & 27.96 & 3.23 & 2.65 & 57.37 & 1.89 & 0.0322 & 2.8359 & 152.98 & 216.68 & 58.16 \\
\hline ED9 & 2.0 & 4.5 & 0.09 & 0.028 & 127.77 & 11.09 & 3.11 & 1.22 & 64.05 & 1.76 & 0.0213 & 1.4676 & 133.91 & 144.63 & 57.76 \\
\hline
\end{tabular}

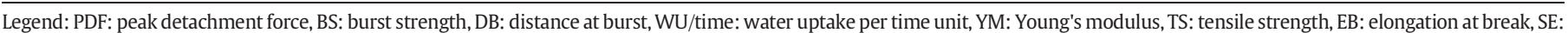
strain energy, $\Delta \mathrm{E}$ : colour difference between each film and the reference film. All data are an average of three measurements, the associated error was always lower than $5 \%$. 

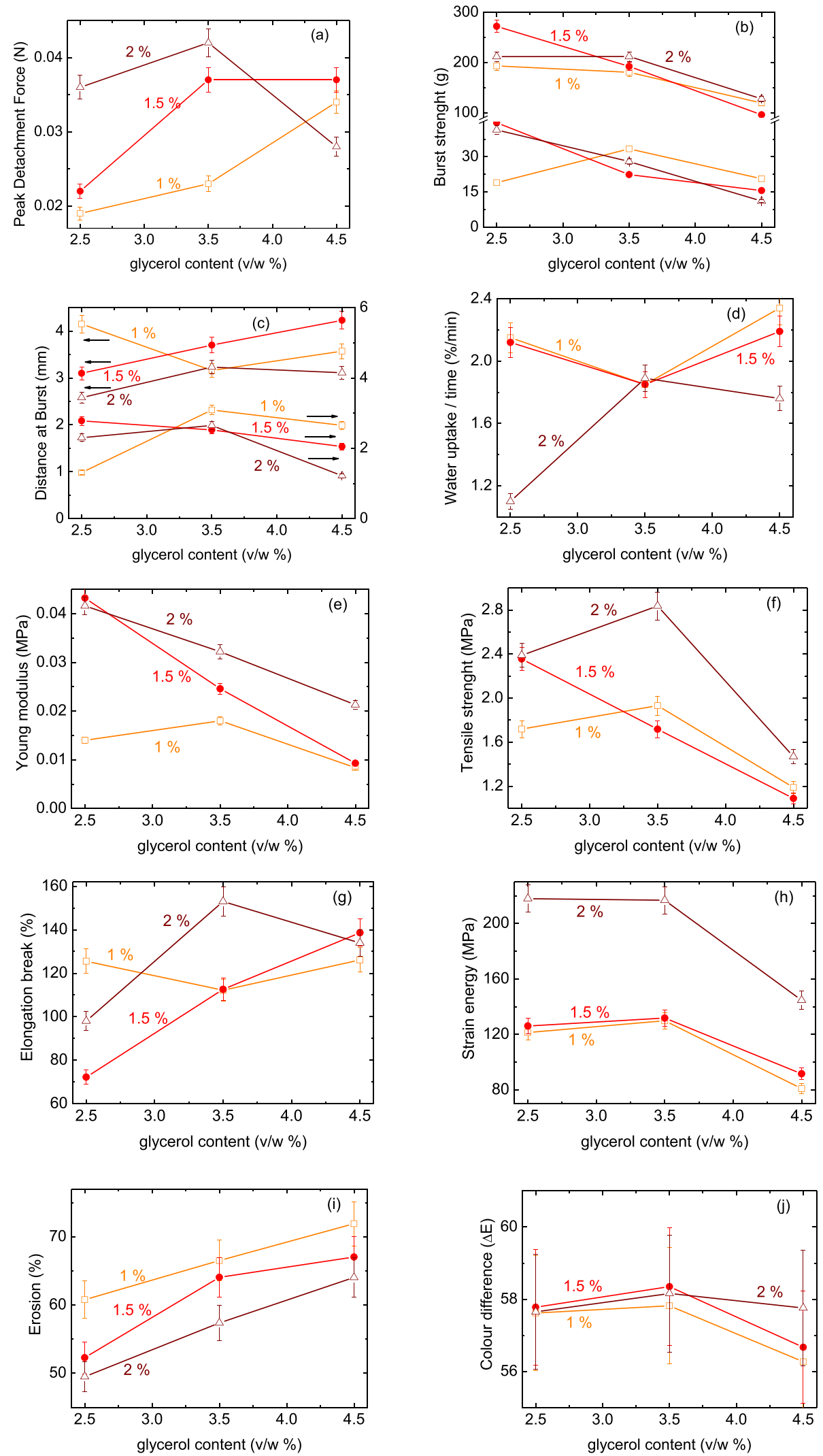

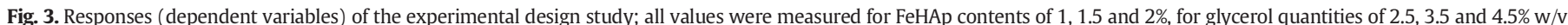

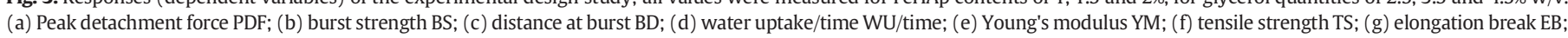
(h) strain energy SE; (i) erosion $\mathrm{E}$; (j) colour difference $\Delta \mathrm{E}$. 
minimised, to obtain the film with the best characteristics. The optimised formulation was found to be a film with $2.55 \% \mathrm{w} / \mathrm{v}$ of glycerol, $2 \% \mathrm{w} / \mathrm{v}$ of FeHAp powder and $0.08 \mathrm{~mm}$ thickness. Table 4 reports the various parameters for the optimised CS-FeHAp film.

These values were compared with those obtained experimentally from films made using these optimised conditions (only $0.08 \mathrm{~mm}$ thick films were measured). It can be seen (Table 4) that there is a very good agreement between predicted and experimental values for all properties considered; this confirms the reproducibility of the sample, as well as the reliability of the model employed for the study.

Literature reports various films and/or membranes for biomedicine prepared with different materials, hence exhibiting different mechanical properties. Because of these differences, a proper comparison is difficult. However, some general comments about the behaviour of the optimised CS-FeHAp film, and its suitability for biomedical use, are possible. Although YM is inferior to other developed membranes, the value is comparable to that of human skin [31]. On the other hand, CS-HAp film shows a better TS performance than a commercial dressing (Kaltostat ${ }^{\circledR}$ ), and comparable values to human skin [32]. CS-FeHAp shows an improved EB performance compared with other chitosan films [33] or commercial dressings [34]. Literature reports CS films swith TS values $\sim 10$ times higher than those observed here [35,36], but film thickness was also 10 times greater. Regarding the PDF, higher values (about 8 times) were obtained for chitosan-based films when additives were included in the matrix $[37,38]$. It has to be highlighted, however, that films with very high bioadhesive properties could stick to the wound and cause damage during the removal [37]. Lower bioadhesion, such as that observed here, can actually be more suitable for wound dressings.

Overall, it can be stated that, although some mechanical properties of the CS-FeHAp film are lower other reported data, the values of such properties are suitable for wound dressing material. Hence, these results indicate that it is possible to obtain films with the required mechanical behaviour and, in addition to this, UV protection as well, making this coating appropriate for use in biomedicine.

\subsection{Functional properties of the optimised film}

Once the conditions for optimised film preparation were established, additional studies of functional properties of the optimised CS-FeHAp film were performed: UPF value, porosity, biocompatibility and antibacterial activity.

\subsubsection{UPF value and photostability}

Measurement of UPF values of optimised CS-FeHAp films confirmed that they can provide very high protection - indeed, the film showed an average UPF value of $\sim 1900$. It can, hence, be classified as excellent. Moreover, the value did not change significantly after the films have been irradiated with UV light for $3 \mathrm{~h}$. This photostability is essential for sunscreen applications.

Table 4

Comparison between the predicted and the experimental values of properties for the optimised film.

\begin{tabular}{lll}
\hline Variable & Predicted value & Experimental value \\
\hline PDF $(\mathrm{N})$ & $0.0431 \pm 0.0212$ & $0.0435 \pm 0.0007$ \\
BS $(\mathrm{g})$ & $174 \pm 53$ & $174 \pm 2$ \\
BS $1 \mathrm{~h}(\mathrm{~g})$ & $34.2 \pm 33.7$ & $34.8 \pm 0.6$ \\
DB $(\mathrm{mm})$ & $3.27 \pm 1.71$ & $3.27 \pm 0.02$ \\
DB $1 \mathrm{~h}(\mathrm{~mm})$ & $2.0 \pm 1.7$ & $2.2 \pm 0.1$ \\
Erosion $(\%)$ & $50.5 \pm 7.2$ & $50.6 \pm 0.3$ \\
WU/time $\left(\% \cdot \mathrm{min}^{-1}\right)$ & $1.13 \pm 1.38$ & $1.17 \pm 0.03$ \\
YM $(\mathrm{MPa})$ & $0.0313 \pm 0.013$ & $0.033 \pm 0.001$ \\
TS $(\mathrm{MPa})$ & $2.31 \pm 1.45$ & $2.36 \pm 0.08$ \\
EB $(\%)$ & $130 \pm 73$ & $133 \pm 4$ \\
SE $(\mathrm{MPa})$ & $230 \pm 19$ & $229 \pm 2$ \\
$\Delta \mathrm{E}$ & $57.37 \pm 1.089$ & $57.38 \pm 0.001$ \\
\hline
\end{tabular}

\subsubsection{Porosity}

Porosity is an important parameter for a wound dressing; indeed, appropriate porosity values can be beneficial the absorption of wound exudates, as well as transfer of nutrients and oxygen to cells [39,40]. The optimised CS-FeHAp films showed a porosity of $53.6 \pm 0.9 \%$. This value is comparable to what reported by Poonguzhali et al. [41], although in other studies chitosan-based films showed higher porosity [39]. It has to be highlighted, however, that porosity can be affected by many parameters, including chitosan degree of reticulation as well as possible additives added to the matrix [41].

\subsubsection{Cytotoxicity}

Fig. 4 shows cytotoxicity test results, keratinocyte viability being shown using cells not in contact with any sample as a positive control reference. This cellular line was chosen as the application of these films would ideally be as wound dressing, so the effect on skin cells such as keratinocytes should be assessed [42,43]. The presence of FeHAp powder in the CS matrix does not have a negative effect on film biocompatibility; indeed, cells cultured with the CS-FeHAp film have viability comparable to that of the positive control, within experimental error.

This indicates that the known and proven biocompatibility of chitosan is not affected by the incorporation of the powder within its structure. Based on these data, it can be stated that CS-FeHAp matrices can be used without any risk to the health of the skin.

\subsubsection{Antibacterial activity}

Fig. 5 shows antimicrobial activity tests of CS-FeHAp films towards three strains, performed to assess whether the presence of the powder incorporated into the film had effected the chitosan's behaviour towards the microbial strains.

Fig. 5a shows the activity towards a Gram-negative strain, E. coli - no significant difference can be observed between the simple CS film and the film containing FeHAp. In both cases, a decrease of almost $2 \log$ units can be observed in the number of the viable bacteria after twohour contact time (1.6-1.7 units, from $>6$ to $\sim 4.5 \log \mathrm{CFU} / \mathrm{mL}$ ), confirming the antibacterial activity of the CS film even when the UVabsorbing powder is incorporated in its matrix. After $4 \mathrm{~h}$ there was no significant change, while after $8 \mathrm{~h}$ a small increase to about $5 \log \mathrm{CFU} /$ swas observed for both samples. Such a small increase indicates a partial recovery of the bacterial strain, as has also been observed for other antibacterial coatings [24]. Despite this increase, however, the bacterial population is still significantly lower than the original value.

Tests with Gram-positive bacterium (MRSA, Fig. 5b) again show that FeHAp in the film does not impact antibacterial activity. MRSA,

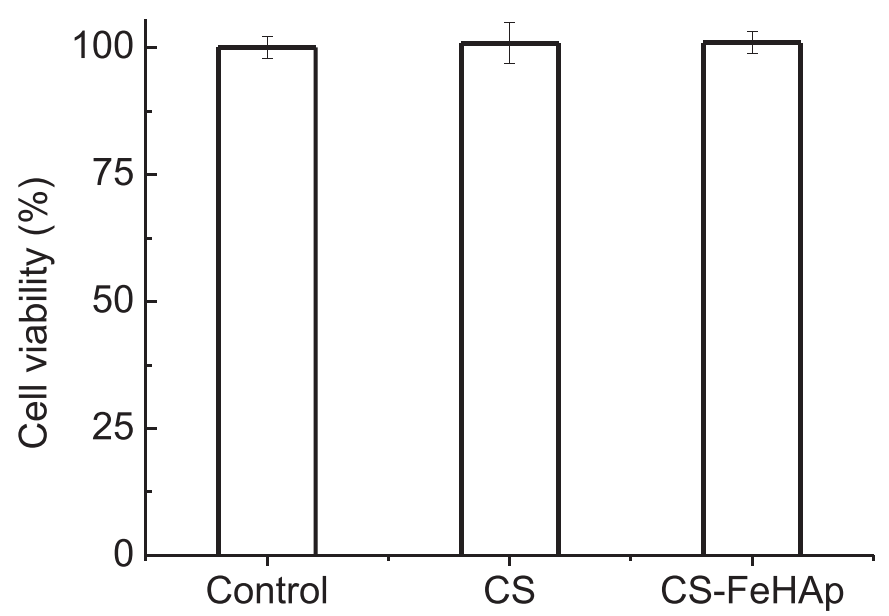

Fig. 4. Viability of keratinocytes cells for the positive control, simple chitosan film (CS) and chitosan with FeHAp powder (CS-FeHAp). The error bars represent the standard deviation of the data, no significant difference was observed between the three samples. 

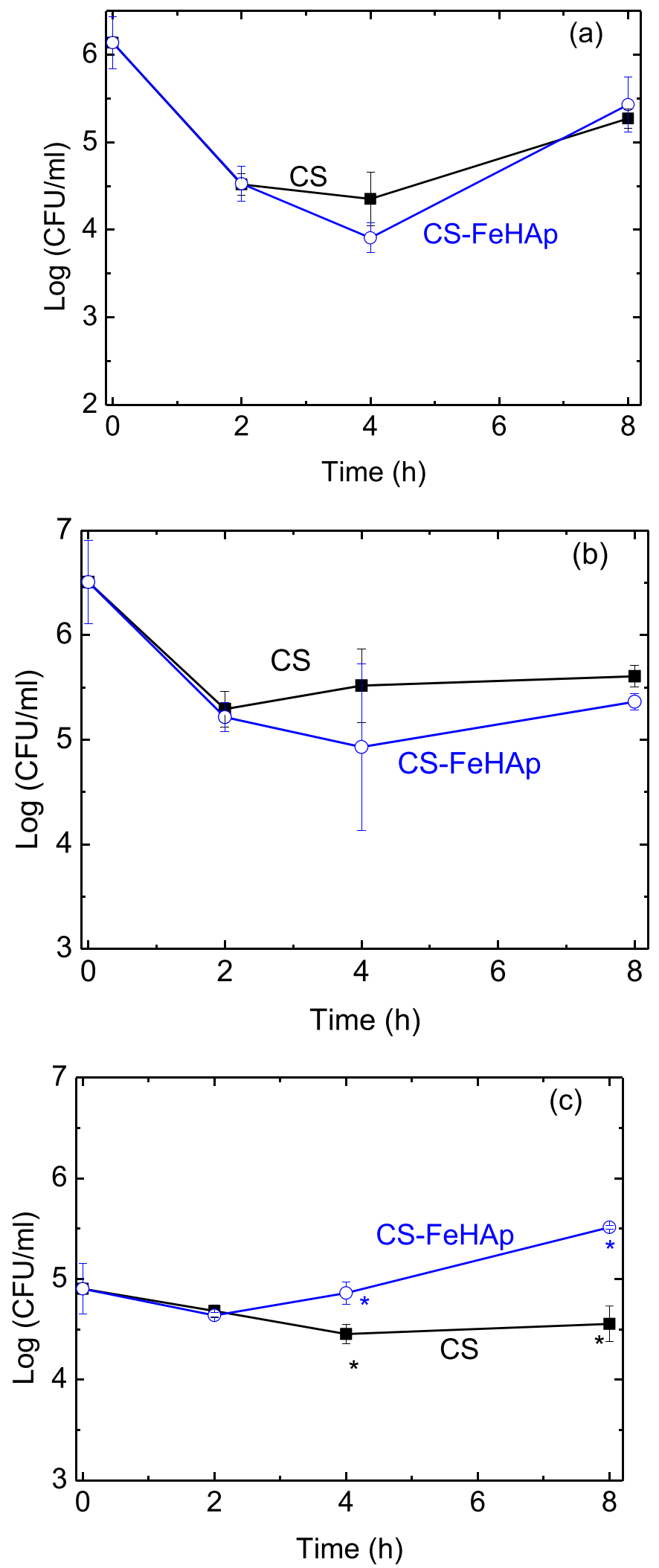

Fig. 5. Bacterial inactivation for films of simple chitosan (CS) and chitosan with FeHAp powder (CS-FeHAp). (a) E. coli; (b) MRSA; (c) Candida albicans. The error bars represent the standard deviation of the data; no significant difference was observed between the data of the different films, apart from Candida albicans at 4 and 8 h (Fig. 3(c), data marked with an asterisk). however, seems to be less susceptible to $\mathbf{C S}$ films, as a decrease of only 1 $\log$ unit was observed. This behaviour towards this Gram-positive strains was previously reported [44].

In tests against Candida albicans (Fig. 5c), CS film does not show significant activity, with a very small decrease $(\sim 0.5 \log$ units $)$ in the active microorganism population. Indeed, CS-FeHAp film seems to promote its growth, and after $8 \mathrm{~h}$ a slight increase in the viable cells was observed. Reduced CS effectiveness towards Candida was already observed in other studies [45]. However, the enhanced growth experienced with CS-FeHAp film is more difficult to explain. It is likely that, during the incubation, compounds favouring fungus growth are released - these could be either organic molecules/fragments acting as a carbon source, or ionic species containing calcium, phosphorus and/or iron, which can have beneficial effects on the strain.

While these substances may have been also released in the tests with E. coli and MRSA, their positive effect may have been masked and/or annulled by the strong antibacterial activity of the CS matrix. Nevertheless, this needs further investigation. On the whole, it could be said that the studied CS-FeHAp films show significant antibacterial effect towards Gram-positive and Gram-negative strains, reaching their highest activity between 2 and $4 \mathrm{~h}$. Although many wound dressings were tested for longer times (i.e. $24 \mathrm{~h}$ ), literature also reports examples of dressings tested for shorter intervals [6]. To maximise their effect, it is advisable to use these films for this length of time, and not longer periods. The CS-FeHAp system, on the other hand, does not seem suitable for inhibiting yeast strains such as Candida $a$. The combination of such antimicrobial activity with the excellent UV-absorbing properties makes this system very suitable as wound dressing.

\section{Conclusions}

Films made of chitosan and Fe-modified HAp of marine origin were successfully prepared. Our hypothesis was confirmed: the addition of FeHAp powder gave additional functionality to the coatings, as they showed excellent UV absorbing properties, indicating that the optical features of the powder was maintained in the coating. At the same time, they retained the properties of un-modified chitosan, such as the antibacterial activity and non-cytotoxicity; their mechanical properties were also good. The combination of all these characteristics makes these films very suitable for biomedical applications as wound dressing, as they can help reduce possible bacterial infection while protecting wounds from UV light exposure.

As future work, in vivo studies will be performed; tests will be aimed to assess wound healing properties (i.e. interaction with plasma, cellular regeneration, proliferation and/or differentiation) as well as to determine the effectiveness of UV protection behaviour.

Combinations of the UV-absorbing powder with other matrices will also be considered.

\section{Acknowledgments}

This work was financially supported by Portuguese government through the project UID/Multi/50016/2013 through the project BioTherapies (Programa Operacional de Desenvolvimento do Norte, No. NORTE-01-0145-FEDER-00012). Clara Piccirillo would like to thank Fondazione con il Sud, to fund the project HApECOrk (no. 2015-0243). Robert Pullar wishes to thank FCT (Fundação para a Ciência e a Tecnologia, Portugal) Grant IF/00681/2015. This work was partly developed within the scope of the project CICECO-Aveiro Institute of Materials, UIDB/50011/2020 \& UIDP/50011/2020, financed by national funds through the FCT/MEC and when appropriate co-financed by FEDER under the PT2020 Partnership Agreement. David Maria Tobaldi is grateful to Portuguese national funds (OE), through FCT, I.P., in the scope of the framework contract foreseen in the numbers 4,5 , and 6 of the article 23, of the Decree-Law 57/2016, of August 29, changed by Law 57/2017, of July 19. 


\section{References}

[1] M. Rinaudo, Chitin and chitosan: properties and applications, Prog. Polym. Sci. 31 (2006) 603-632.

[2] E.M. Costa, S. Silva, S. Vicente, C. Neto, P.M. Castro, M. Veiga, R. Madureira, F. Tavaria M.M. Pintado, Chitosan nanoparticles as alternative anti-staphylococci agents: bactericidal, antibiofilm and antiadhesive effects, Mater. Sci. Eng. C 79 (2017) 221-226.

[3] S.P. Miguel, A.F. Moreira, I.J. Correia, Chitosan based-asymmetric membranes for wound healing: a review, Int. J. Biol. Macromol. 127 (2019) 460-475.

[4] B. Gerber, P. Mathys, M. Moser, D. Bressoud, C. Braun-Fahrländer, Ultraviolet emission spectra of sunbeds, Photochem. Photobiol. 76 (2007) 664-668.

[5] H.M. Rogers, M.A. Weinstock, S.R. Feldman, B.M. Coldiron, Incidence estimate of non-melanoma skin cancer (keratinocyte carcinomas) in the US population, 2012, JAMA Dermat 151 (2015) 1081-1086.

[6] S. Jiang, B.C. Ma, J. Reinholz, Q. Li, J. Wang, K.A.I. Zhang, K. Lanfester, D. Crespy, Efficient nanofibrous membranes for antibacterial wound dressing and UV protection, ACS Appl. Mater. Interf. 8 (2016) 29915-29922.

[7] A. Gupta, P. Avci, T. Dai, Y.Y. Huang, M.R. Hamblin, Ultraviolet radiation in wound care: sterilization and stimulation, Adv. Wound Care 2 (2013) 422-437.

[8] S.C.M. Fernandes, A. Alonso-Valona, T. Palomares, V. Zubillaga, J. Labidi, V. Bulone, Exploiting mycosporines as natural molecular sunscreens for the fabrication of UV-absorbing green materials, ACS Appl. Mater. Interf. 7 (2015) 16658-16664.

[9] Y.A. Gomaa, L.K. El-Khordagui, N.A. Boraei, I.A. Darwish, Chitosan microparticles incorporating a hydrophilic sunscreen agent, Carb. Polym. 81 (2010) 234-242.

[10] L. Wongkom, A. Jimtaisong, Novel biocomposite of carboxymethyl chitosan and pineapple peel carboxymethylcellulose as sunscreen carrier, Int. J. Biol. Macromol. 95 (2017) 873-880.

[11] C. Piccirillo, R.C. Pullar, E. Costa, A. Santos Silva, M.M. E. Pintado, P.M. L. Castro, Hydroxyapatite-based materials of marine origin: a bioactivity and sintering study, Mater. Sci. Eng. C 51 (2015) 309-315.

[12] T.S. de Araujo, S.O. de Souza, W. Miyakawa, E.M.B. de Sousa, Phosphates nanoparticles doped with zinc and manganese for sunscreens, Mater. Chem. Phys. 124 (2010) 1071-1076.

[13] C. Piccirillo, C. Rocha, D.M. Tobaldi, R.C. Pullar, J.A. Labrincha, M.O. Ferreira, P.M.L Castro, M.M.E. Pintado, A hydroxyapatite- $\mathrm{Fe}_{2} \mathrm{O}_{3}$ based material of natural origin as an active sunscreen, J. Mater. Chem. B 2 (2014) 5999-6009.

[14] L. Pighinelli, M. Kucharska, Chitosan-hydroxyapatite composites, Carb. Polym. 93 (2013) 256-262.

[15] R. Morsy, S.S. Ali, M. El-Shetehy, Development of hydroxyapatite-chitosan gel sunscreen combating clinical multi-drug resistant bacteria, J. Mol. Str. 1143 (2017) 251-260.

[16] M. Rincón-Fontán, L. Rodríguez-López, X. Vecino, J.M. Cruz, A.B. Moldes, Design and characterization of greener sunscreen formulations based on mica powder and biosurfactant extract, Powder Tech 327 (2018) 442-448.

[17] M.A.C. Teixeira, C. Piccirillo, D.M. Tobaldi, R.C. Pullar, J.A. Labrincha, M.O. Ferreira, P.M.L. Castro, M.M.E. Pintado, Effect of preparation and processing conditions on UV absorbing properties of hydroxyapatite- $\mathrm{Fe}_{2} \mathrm{O}_{3}$ sunscreens, Mater. Sci. Eng. C 71 (2017) 141-149.

[18] Standards Australia/New Zealand Standard, AS/NZS, 4399:1996, Sun Protective Clothing - Evaluation and Classification, Homebush NSW/Wellington, Standards Australia/ Standards New Zealand, 1996https://shop.standards.govt.nz/catalog/ 4399:2017(AS\%7CNZS)/scope.

[19] P. Cazón, P. Vásquez, G. Velasquez, Composite films of regenerate cellulose with chitosan and polyvinyl alcohol: evaluation of water adsorption, mechanical and optical properties, Int. J. Biol. Macrom. 117 (2018) 235-246.

[20] U. Kalapathy, A. Proctor, J. Shults, A simple method for production of pure silica from rice hull ash, Bioresour. Technol. 73 (2000) 257-262.

[21] A.A. Mahmoud, A.H. Salama, Norfloxacin-loaded collagen/chitosan scaffolds for skin reconstruction: preparation, evaluation and in-vivo wound healing assessment, Europ. J. Pharm. Sci. 83 (2016) 155-165.

[22] M.T. Khorasani, A. Joorabloo, H. Adeli, Z. Mansoori-Moghadam, A. Moghaddam, Design and optimization of process parameters of polyvinyl (alcohol)/chitosan/nano zinc oxide hydrogels as wound healing materials, Carbohydr. Polym. 207 (2019) $542-554$.

[23] ISO document 10993-5 I, Biological Evaluation of Medical Devices Test for in Vitro Cytotoxicity, International Organization for Standardization, International Organization for Standardization, Geneva, Switzerland, 1999.
[24] D. Campos, C. Piccirillo, R.C. Pullar, P.M.L. Castro, M.M.E. Pintado, Characterization and antimicrobial properties of food packaging methylcellulose films containing stem extract of Ginja cherry, J. Sci. Food Agric. 94 (2014) 2097-2103.

[25] R. Thakur, B. Saberi, P. Pristijono, C.E. Stathopoulos, J.B. Golding, C.G. Scarlett, M. Bowyer, Q.V. Vuong, Use of response surface methodology (RSM) to optimize pea starch-chitosan novel edible film formulation, J. Food Sci. Tech. 54 (2017) 2270-2278.

[26] J.A. Sirviö, M. Visanko, J. Ukkola, H. Liimatainen, Effect of plasticizers on the mechanical and thermomechanical properties of cellusose-based biocomposite films, Ind. Crop. Prod. 122 (2018) 513-521.

[27] E.M. Rivera-Muñoz, G.M. Raucci, G. Buonocore, Chitosan-hydroxyapatite nanocomposites: effect of interfacial layer on mechanical and dielectric properties, Mater. Chem. Phys. 217 (2018) 151-159.

[28] S. Prateepchanachai, W. Thanhiew, S. Devahastin, S. Soponronnarit, Mechanical properties improvement of chitosan films via use of plasticizer, charge modifying agent and film solution homogeneization, Carb. Polym. 174 (2017) 253-261.

[29] M. Xie, K. Huang, F. Yang, R. Wang, L. Han, H. Yu, Z. Ye, F. Wu, Chitosan nanocomposite films based on halloysite nanotubes modification for potential biomedical applications, Int. J. Biol. Macrom. 151 (2020) 1116-1125.

[30] Y. Xu, Y. Chu, X. Feng, C. Gao, D. Wu, W. Cheng, L. Meng, Y. Zhang, X. Tang, Effects of zein stabilized clove essential oil Pickering emulsion on the structure and properties of chitosan-based edible films, Int. J. Biol. Macrom. 156 (2020) 111-119.

[31] Y. Yang, L. Qiu, L. Wang, X. Xiang, Y. Tang, H. Li, F. Yan, Quantitative assessment of skin stiffness using ultrasound shear wave elastography in systemic sclerosis, Ultras. Medic. Biol. 45 (2019) 902-912.

[32] R. Gharibi, S. Kazemi, H. Yeganeh, V. Tafakori, Utilizing dextran to improve hemocompatibility of antimicrobial wound dressing with embedded quaternary ammonium salts, Int. J. Biol. Macrom. 131 (2019) 1044-1056.

[33] P.K. Mandapalli, S. Labala, S. Chawla, R. Janupalli, D. Sriram, V.V.K. Venuganti, Polymer-gold nanoparticle composite films for topical application: evaluation of physical properties and antibacterial activity, Polym. Compos. 38 (2015) 2829-2840.

[34] J.J. Elsner, A. Shefy-Peleg, M. Zilberman, Novel biodegradable composite wound dressing with controlled release of antibiotics: microstructure, mechanical and physical properties, J. Biomater. Appl. B 93 (B) (2010) 425-435.

[35] Z Shariatinia, Z Zahraee, Controlled release of metformin from chitosan-based nanocomposite films containing mesoporous MCM-41 nanoparticles as novel drug delivery systems, J. Coll. Interf. Sci. 501 (2017) 60-76.

[36] H. Wang, X. Gong, Y. Miao, X. Guo, C. Liu, Y.Y. Fan, J. Zhang, B. Niu, W. Li, Preparation and characterization of multilayer films composed of chitosan, sodium alginate and carboxymethyl chitosan-ZnO nanoparticles, Food Chem. 283 (2019) 397-403.

[37] X.Y. Chen, H.R. Low, X.Y. Loi, L. Merel, M.A.M.C. Iqbal, Fabrication and evaluation of bacterial nanocellulose/poly(acrylic acid)/ graphene oxide composite hydrogel: characterizations and biocompatibility studies for wound dressing, J. Biomed. Mater. Res. B (2019)https://doi.org/10.1002/jbm.b.34309.

[38] V.A.M. Gonzaga, A.L. Poli, J.S. Gabriel, D.Y. Tezuka, T.A. Valdes, A. Leitão, C.F. Rodero, T.M. Baubab, M. Chorilli, C.C. Schmidtt, Chitosan-laponite nanocomposite scaffolds for wound dressing application, J. Biomed. Mater. Res. (2019)https://doi.org/10. $1002 / j b m . b .34487$.

[39] K. Shanmugapriya, H. Kim, P.S. Saravana, B.S. Chun, H.W. Kang, Fabrication of multifunctional chitosan-based nanocomposite film with rapid healing and antibacterial effect for wound management, Int. J. Biol. Macrom. 114 (2018) 204-213.

[40] V.A.M. Gonzaga, A.L. Poli, J.S. Gabriel, D.Y. Tezuka, T.A. Valdes, A. Leitão, C.F. Rodero, T.M. Baubab, M. Chorilli, C.C. Schmitt, Chitosan-laponite nanocomposite scaffolds for wound dressing application, J. Biomed. Mater. Res. 108 (2020) 1388-1397.

[41] R. Poonguzhali, S. Khaleel Basha, V. Suganta Kumari, Fabrication of asymmetric nanostarch reinforced chitosan/PVP membrane and its evaluation as an antibacterial patch for in vivo would application, Int. J. Biol. Macrom. 118 (2018) 1713-1725

[42] C. Wiegand, U.C. Hipler, Evaluation of biocompatibility and cytotoxicity using keratinocyte and fibroblast cultures, Skin Pharm. Phys. 22 (2009) 74-82.

[43] R. Esteban-Vives, M.T. Young, J. Ziembicki, A. Corcos, J.C. Gerlach, Effects of wound dressing on cultured primary keratinocytes, Burns 42 (2016) 81-90.

[44] A.KT. Chang, R.R. Frias, LV. Alvarez, U.G. Bigol, J.P.M.D. Guzman, Comparative antibacterial activity of commercial chitosan and chitosan extracted from Auricularia sp, Biocat. Agric. Biotech. 17 (2019) 189-195.

[45] N.A. Kamel, S.L. Abd El-messieh, N.M. Saleh, Chitosan/banana peel powder nanocomposites for wound dressing application: preparation and characterization, Mater. Sci. Eng. C 72 (2017) 543-550. 